\title{
La problemática del patrimonio digital: El caso de Costa Rica
}

\author{
José Bernal Rivas Fernández \\ Universidad de Costa Rica - UCR. Costa Rica
}

ANÁLISIS / ANALYSIS

\begin{abstract}
Resumen
En este artículo se analiza la problemática a la que se ve enfrentada la producción de documentos en soporte digital, a raíz de las últimas tendencias en cuanto al gobierno electrónico que plantea nuevos retos para los archivos y para el acceso a la información contenida en este tipo de soporte. Para ello se hace un breve repaso al caso de Costa Rica, donde ya se han producido algunos efectos en cuanto a la protección del patrimonio digital especialmente por el impacto de las tecnologías de la información y la comunicación.
\end{abstract}

Palabras clave

Archivos ; Tecnologías de la información y la comunicación ; Acceso a la información ; Gobierno electrónico

\section{The problem of digital heritage: the case of Costa Rica}

\begin{abstract}
In this article we analyze the problem you are facing the production of documents in digital form, as a result of the latest trends in the e-government that poses new challenges for archives and access to information contained in this type of support. This is a brief review the case of Costa Rica, where there have already been some effects in terms of the protection of digital heritage especially by the impact of information technologies and communication.
\end{abstract}

Keywords

Archives ; Information technologies and communication ; Access to information ; E-Government

\section{Introducción}

El uso intensivo de las tecnologías de la información y la comunicación dentro del marco de la sociedad de la información ha tenido un gran impacto no solo en el mundo de los archivos sino también en el ámbito de la administración pública, planteando problemas en la forma en que se producen los documentos, en cómo se tratan, en cómo se conservan y en cómo se facilita el acceso a la información en ellos contenida, particularmente en cuanto al soporte utilizado y en especial en cuanto al patrimonio digital. Por lo que es necesario reflexionar sobre esta situación de repercusión mundial pero que también ha producido efectos en el caso de Costa Rica, esta problemática tiene distintas aristas, por lo que solamente nos ocuparemos de las iniciativas planteadas a nivel gubernamental, con la emisión de alguna normativa relacionada con el tratamiento de los archivos, la implementación del gobierno electrónico, la puesta en práctica de la firma digital, entre otros temas. 


\section{Los archivos}

Los archivos no son importantes solamente desde el punto de vista cultural, sino también desde la perspectiva de las prácticas para una gestión eficiente. Las buenas prácticas en la gestión de los archivos son una condición para asegurar la continuidad, la eficiencia y la transparencia de los servicios públicos y privados. La rendición de cuentas por parte de las instituciones gubernamentales se fortalece poniendo atención constante en la buena gestión de los archivos, fundamento de la democracia y del derecho a la información. En la sociedad de la información, el significado de los archivos trasciende las fronteras nacionales; por lo que conceptos relacionados como "records keeping" y accesibilidad necesitan ser reconsiderados.

En la época actual es importante preguntarse, ¿es el archivo un lugar de custodia y servicio de los documentos? Al hacer un análisis de la evolución del concepto y funciones del archivo, se concluye que es también "centro de información". El archivo como centro de información cumple su tarea informativa, en principio, en cuanto difunde hacia el exterior sus fondos y su última finalidad es proporcionar información, aunque sus fines inmediatos son recoger, custodiar y servir los documentos (Alvarez, 2007, p.12).

El archivo, concebido como centro de custodia de documentos generados por las instituciones en el desarrollo de sus actividades, con la obligación de ponerlos al servicio del ciudadano y de la sociedad en general, tal y como la indica la Archivística, ha derivado en lo que nos indica la doctora Antonia Heredia, en la "Administración de Archivos". Esta "nueva" concepción del archivo, que traslada su origen al momento en que se genera el documento, determina su integración en la institución, formando parte de un sistema que involucra todo el ciclo de vida de los documentos, conformando las series documentales.

Por lo tanto, los archivos han dejado de ser centros elitistas para uso de unos pocos y con fines prioritarios de investigación y cultura, para convertirse en centros de custodia y gestión de fondos documentales al servicio de la institución productora y por tanto, de los ciudadanos. En el caso de los archivos públicos, además, deben ser soporte de transparencia administrativa y de una gestión pública democrática, capaz de garantizar los derechos.

En el ayer y en el hoy, no hay decisión alguna de la administración que no genere documentos dado el carácter de la expresión institucional de la misma, los archivos están íntimamente ligados a la dimensión esencial de la acción del gobierno y corren a la par de las políticas cambiantes. Por eso en cierta medida, la historia de los archivos es el reflejo de la historia del Estado, del poder de la administración pública y privada. A este propósito conviene recordar que la interpretación posmodernista de los documentos de archivo como fuentes de la historia hace tanto énfasis en los testimonios, como en los silencios y vacíos de los mismos, pues estos hablan tanto como aquellos.

Esto hasta ahora. Pero, ¿cuál debe ser el papel del archivo ante el gran reto de la sociedad de la información, la administración electrónica y los "documentos en diferentes soportes"? La perspectiva cambia sobremanera. De manera tal que es importante tener claridad en cuanto a que estamos entendiendo por tales conceptos (Alvarez, 2007, p.13).

En efecto, los archivos, los repositorios documentales que conservan patrimonio documental, para llegar a organizarlos, hoy se debe hacer, desde los archivos de gestión, los documentos son propios de la naturaleza institucional de la administración pública que por su propia condición son consubstánciales a las mismas cuyas partes se articulan mediante sistemas de administración y gestión documental; son parte legítima y necesaria para el funcionamiento del Estado como ordenador de la vida social; constituyen herramienta indispensable para la administración de la justicia que es la suprema razón de la existencia de aquel y como centros de información contribuyen a la eficacia, transparencia y secuencia de las actividades públicas y privadas (Alvarez, 2007, p.4).

En la sociedad actual, los archivos cumplen importantes cometidos que conllevan desde la necesidad de conservarlos por ser depositarios de la memoria de los pueblos, hasta la de explotarlos como fuentes de información que deben ponerse al alcance de los ciudadanos en la sociedad de la información. Dar cumplimiento a dichos cometidos, aparentemente antagónicos e irreconciliables, no es en la actualidad un asunto insalvable, al menos no por la existencia de las tecnologías de la información y la comunicación (Añorve, 2007, p. 128).

En el caso de los archivos públicos, concentran la documentación de las actuaciones del Estado en sus diferentes sectores y ámbitos del gobierno. En ellos se sedimenta la gestión documental, como consecuencia de la propia actuación de las organizaciones en el cumplimiento de las funciones que se le ha encomendado. De este modo, 
constituyen la huella de gestión del Estado, por lo que de ellos se puede extraer el sustento del trabajo de los archivistas, lo cual nos permitirá determinar la buena o mala actuación que se ha realizado a lo largo del tiempo.

La gestión documental también es cultura, cultura organizativa, es preparar el patrimonio de nuestro futuro pero sobretodo es mejora en las organizaciones, mejora en su rendimiento, en su organización interior, en su estructura de producción, lo que puede llevar a cabo mediante la implementación de un programa de gestión documental.

Un programa de gestión documental se puede contextualizar como el conjunto de instrucciones en las que se detallan las operaciones para el desarrollo de los procesos al interior de cada entidad, tales como la producción, recepción, distribución, trámite, organización, consulta, conservación y disposición final de los documentos. Con urgencia, hay que ir aplicando los programas de gestión documental, para organizar los documentos de los archivos; sin embargo algunos teóricos recomiendan considerar, entre otras cosas: aspectos administrativos es decir situaciones administrativas de la gestión de documentos en aspectos como la transparencia, la simplificación de trámites y la eficiencia de la administración y aspectos económicos en donde el análisis de situaciones de tipo económico, como la reducción de costos derivados de la conservación de documentos innecesarios y la racionalización de los recursos destinados para la gestión documental. Con el diseño de programas de gestión documental se buscan objetivos como:

Resaltar la importancia del papel de los documentos y archivos como lenguaje natural de la administración para el funcionamiento de la misma, elementos necesarios para la participación ciudadana, apoyos decisivos para la transparencia y el control de la gestión así como garantía de derechos individuales y colectivos. Procurar la racionalización y control de la producción documental, en atención a los procedimientos, trámites administrativos y flujos documentales, lo mismo que, la normalización de modelos y formatos para la producción documental.

Normalizar la utilización de materiales, soportes y equipos de calidad y que a la vez preserven el medio ambiente.

a) Integrar el servicio de recepción de comunicaciones oficiales con el archivo, para optimizar los procesos de organización de los documentos, en clasificación, ordenación y descripción.

b) Aplicar normas homologadas en descripción documental, como la ISAD (G) e ISAAR (CPF).

c) Facilitar el diseño de servicios y mercadeo de la información en forma oportuna. la conservación de documentos innecesarios y la racionalización de los recursos destinados para la gestión documental; y aspectos archivísticos considerando elementos básicos como el ciclo de vida de los documentos, los principios de procedencia y de orden original, la clasificación, descripción basada en normas internacionales y la incorporación de las TIC en el manejo de los documentos" (Alvarez, 2007, p.7-8).

La disponibilidad de las tecnologías de la información ha facilitado enormemente la accesibilidad a la información, transformando por completo las estructuras organizativas, así como la manera de relacionarse los individuos, tanto entre ellos mismos como con las administraciones. Con esta concepción globalizadora y unitaria para la organización de los documentos administrativos en su ciclo de vida, unido a la complejidad de las administraciones tanto en su organización como en sus métodos de trabajo -que se constata en la producción, circulación y uso de los documentos, los profesionales de la Archivística deben tomar conciencia de la necesidad de una gestión moderna de los documentos.

\section{Las tecnologías de la información y la comunicación}

Las tecnologías de la información y la comunicación (TIC) podrían definirse como un conjunto de herramientas e infraestructuras utilizadas para la recogida, almacenamiento, tratamiento, difusión y transmisión de la información. Son herramientas con (un enorme) potencial para configurar estructuras organizativas y modelos de gestión pública que ofrezcan una respuesta única, ágil, eficiente, de calidad y transparente a los ciudadanos. De esta manera la extensión y el uso de las TIC y la creación de iniciativas y proyectos de gobierno electrónico, -como anteriormente pudieron serlo las ideas de receptividad de la Administración, orientación al ciudadano, calidad y mejora continua en 
la prestación de servicios-, podrían ser un motor de cambio para las organizaciones, existiendo también la amenaza de que sea o se convierta en una nueva moda, (Pocoví y Farabollini, 2002, p.3).

En el contexto de la Era Digital, las tecnologías de la información y las comunicaciones electrónicas permiten la creación y explotación de un ciclo de gestión de la información más disponible y eficaz, de manera que ésta puede ser creada, organizada, difundida, conservada y puesta a disposición de los ciudadanos. De tal modo que confluyen factores jurídicos, políticos, económicos, tecnológicos y de organización de la información en el proyecto de poner a disposición de los ciudadanos dicha información. Esta evidencia de que la información pública se ha convertido en un gran recurso económico es paralela al desarrollo de la sociedad de la información, aunque algunos encuentran antecedentes más lejanos (Ramos, 2009, p.44).

En los últimos años, el desarrollo y la extensión del uso de las tecnologías de la información y la comunicación (TIC) y la promoción de la sociedad de la información se han convertido en una prioridad política. Ello se pone de manifiesto en documentos e informes procedentes de organizaciones internacionales (OCDE,1998; G-8, 1998) o supra-nacionales (Comisión Europea, 1994, 1997); así como en proyectos e iniciativas comunitarias (e-Europe), estatales (Info XXI en España; Portugal Digital; IS for all in Denmark; Finland as an IS) o planes regionales (Cataluña, Canarias, País Vasco, Extremadura, Murcia...) que recomiendan a los diferentes niveles de gobierno el uso, promoción y universalización de las TIC para: 1) posibilitar el desarrollo de una economía basada en el conocimiento que fomente la inclusión social y disminuya las desigualdades en el acceso y uso de las TIC; y para 2) garantizar la receptividad de los Gobiernos y Administraciones Públicas y una gestión de los recursos públicos no sólo más eficaz y eficiente, sino más democrática y participativa, que responda a las necesidades de los ciudadanos (Plan de Acción INFO XXI, 2001.2). (Criado Grande; Ramilo Araujo y Salvador Serna, 2002, p. 2).

La introducción de las TIC en las organizaciones públicas constituye una de las principales transformaciones de los estados contemporáneos. En el análisis de estas dinámicas de cambio suele destacarse su impacto tanto sobre el sistema político, habitualmente asociado a un supuesto incremento del grado de democratización, como sobre el sistema administrativo, destacándose la transformación en el funcionamiento interno y en las dinámicas de relación con la sociedad.p.19 Los argumentos a favor de la introducción de las TIC en las actividades de los gobiernos vienen a destacar su potencial para mejorar la eficiencia, la calidad y la responsabilidad del sector público (Pocoví y Farabollini, 2002, p.1).

La tecnología de información debe ser concebida en forma amplia para encauzar la creación y uso de la información organizacional. El beneficio de la sola automatización de funciones es de tipo táctico, de corto plazo y lleva a la creación de "islas automatizadas de información". El aporte que debe proveer la TI es en función de la integración y se debe alinear con la estrategia global de la organización.

Las nuevas tecnologías e Internet mejoran la difusión en los siguientes aspectos:

- Facilitan el acceso de los ciudadanos a la información proporcionada por el sector público.

- Favorecen el intercambio de información entre instituciones.

- Propician un uso de la información con fines privados, incluida su explotación comercial.

- El acceso y la gestión de esa información por medios electrónicos fuera del contexto espaciotemporal plantea a las autoridades públicas desafíos jurídicos, económicos, técnicos. (¿qué es, cuánto cuesta, tienen que tener todos los ciudadanos acceso a las nuevas tecnologías informativas, es necesario mantener también las formas de acceso tradicionales?). (Ramos, 2009, p. 47)

El tema de las TIC"s debe enmarcarse dentro del contexto de la sociedad de la información (SI) que recoge la necesidad de plantear políticas públicas de desarrollo, se trata de una definición basada en el concepto de virtualidad real, o lo que es lo mismo, la idea según la cual las TIC permiten una interconexión e identificación de la información, gracias al hipertexto, desde cualquier lugar del planeta, de manera instantánea. Dada la multiplicidad de significados que se atribuyen a la SI, y ya que no se ha impuesto una definición comúnmente aceptada, en este ensayo entendemos por SI un estadio de desarrollo social caracterizado por la capacidad de sus miembros 
(ciudadanos, empresas y Administración Pública) para obtener y compartir cualquier información, instantáneamente, desde cualquier lugar y en la forma que se prefiera'. El concepto SI se refiere a la creciente importancia que han ido adquiriendo la información y la comunicación desde la década de los setenta del siglo XX.

Hablar de sociedad de la información es hablar de una era en la que la interacción humano-tecnología acapara todos los espacios, tiempos, actividades y hábitos de la vida cotidiana. Su origen se remonta a los años setenta cuando comenzó a percibirse que la sociedad industrial empezaba a evolucionar a un tipo de sociedad distinta, en el que el control y optimización de los procesos industriales, y movimientos económicos eran remplazados por el proceso y manejo de la Información. "Sociedad de la Información es una fase de desarrollo social caracterizada por la capacidad de sus miembros para obtener y compartir cualquier información instantáneamente, desde cualquier lugar y en la forma que se prefiera" (González, 2005, p.532).

El factor diferencial que introduce la sociedad de la información es que cada persona u organización no solo dispone de sus propios almacenes de conocimiento, sino que tiene también una capacidad casi ilimitada para acceder a la información generada por los demás, y el potencial para convertirse el mismo en un generador de información para otros. Este cambio en la capacidad de acceso a la Información es tan importante que constituye el factor desencadenante de una serie de transformaciones sociales de profundo alcance (González, 2005, p.539).

\section{El patrimonio digital}

El patrimonio digital consiste en recursos únicos que son fruto del saber o la expresión de los seres humanos. Comprende recursos de carácter cultural, educativo, científico o administrativo e información técnica, jurídica, médica y de otras clases, que se generan directamente en formato digital o se convierten a éste a partir de material analógico ya existente. Los productos "de origen digital" no existen en otro formato que el electrónico (Carta Patrimonio Digital, 2003, p.80).

Los objetos digitales pueden ser textos, bases de datos, imágenes fijas o en movimiento, grabaciones sonoras, material gráfico, programas informáticos o páginas Web, entre otros muchos formatos posibles dentro de un vasto repertorio de diversidad creciente. A menudo son efímeros, y su conservación requiere un trabajo específico en este sentido en los procesos de producción, mantenimiento y gestión. Muchos de esos recursos revisten valor e importancia duraderos, y constituyen por ello un patrimonio digno de protección y conservación en beneficio de las generaciones actuales $y$ futuras.

El objetivo de la conservación del patrimonio digital es que éste sea accesible para el público. Por consiguiente, el acceso a los elementos del patrimonio digital, especialmente los de dominio público, no debería estar sujeto a requisitos poco razonables. Además, el patrimonio digital del mundo corre el peligro de perderse para la posteridad. Contribuyen a ello, entre otros factores, la rápida obsolescencia de los equipos y programas informáticos que le dan vida, las incertidumbres existentes en torno a los recursos, la responsabilidad y los métodos para su mantenimiento y conservación y la falta de legislación que ampare estos procesos (Carta Patrimonio Digital, 2003, p.80).

La evolución de la tecnología digital ha sido tan rápida y onerosa que los gobiernos e instituciones no han podido elaborar estrategias de conservación, oportunas y bien fundamentadas, como analizaremos más adelante. No se ha comprendido en toda su magnitud la amenaza que pesa sobre el potencial económico, social, intelectual y cultural que encierra el patrimonio, sobre el cual se edifica el porvenir.

El primer tipo de cuestiones, relacionado con el mantenimiento de cada objeto digital, puede parecer fácil de resolver utilizando la migración. Sin embargo, la migración de entidades digitales que interactúan y que existen en diferentes formatos a menudo hace que su interacción sea imposible, altera la funcionalidad de las entidades, y da como resultado reproducciones parciales, inexactas, poco fiables y no auténticas. La dificultad del segundo tipo de cuestiones, relacionado con el mantenimiento de las relaciones entre entidades analógicas y digitales, se deriva del hecho de que esas relaciones, así como la interacción entre las entidades digitales en cuestión, no están usualmente documentadas de un modo que haga posible volver a representarlas en un entorno diferente, o cuando una o más de las entidades digitales se actualiza. 
Para cada servicio proporcionado digitalmente por un gobierno a un ciudadano de modo interactivo, existirá una dispersión del documento archivístico entre varias tecnologías en interacción, un documento archivístico que no tiene límites claros, y que cambia continuamente sobre la base del input del usuario (o el funcionario o el ciudadano) y/o de la reacción del sistema a este input; y un documento archivístico que raramente se corresponde con una acción, y que muy a menudo incluye toda la interacción entre una oficina del gobierno y un ciudadano con respecto a un asunto (esto es, lo que solía ser un expediente en papel). Así, pues, habrá que identificar: 1) los límites de la entidad digital que constituye el documento archivístico que, una vez realizado o recibido, y guardado repetidamente en diferentes instancias, será conservado para acción o referencia futura en un sistema fiable de conservación de documentos archivísticos, 2) la esencia de tal entidad, esto es, teniendo en mente el grado aceptable de cambio desde el momento en que el documento archivístico ha logrado su instalación final, sus partes constituyentes y componentes digitales, a ser conservados estables en cuanto contenido y fijos en cuanto forma, y enlazados entre ellos, 3) sus atributos a ser manifestados en los metadatos permanentemente adjuntos al documento archivístico como una anotación (convirtiéndose por tanto en una parte constituyente del documento archivístico), y 4) la documentación complementaria necesaria de lo que no sea completamente conservable, esto es, interactividad, conectividad y funcionalidad. Una vez que esta identificación ha tenido lugar, se podría ensamblar la esencia estabilizada del documento archivístico, sus metadatos y la documentación del sistema, y tratar esta entidad como el documento archivístico. ¿Sería aceptable tal procedimiento si fuera interés y responsabilidad del creador el ejecutarlo? ¿Es una idea tal contraria a la comprensión teórica de lo que es un documento archivístico?

Como uno de los requisitos técnicos en los inicios del gobierno electrónico, la implementación del documento digitalizado y su masificación a través de la automatización de oficinas, puede implicar enormes ventajas y ahorros que seguramente superan las expectativas. Sin embargo, su implementación en la administración pública requiere una serie de transformaciones que no pasan solamente por lo tecnológico y lo legal-normativo. El tendido de redes de comunicación de datos se debe hacer con un objetivo mucho más ambicioso que conectar las computadoras personales, en el marco formal de una nueva cultura de la información (Pocoví y Farabollini, 2002. p.14).

En este escenario, se deben aprovechar las ventajas del flujo de información digital para reducir gastos, mejorar procesos y la calidad de los servicios; posibilitando al gobierno la interacción electrónica con los ciudadanos, sus proveedores y entre sus funcionarios, haciendo más transparentes los procesos, contra la corrupción. Esto permite orientar la gestión hacia la comunidad, multiplicar la conexión de usuarios de TIC y expandir ampliamente la provisión de nuevos servicios, debiendo asegurar facilidades de acceso que disminuyan la "brecha digital".

\section{El gobierno electrónico}

El gobierno electrónico es un fenómeno relativamente reciente que no ha sido claramente definido. Representa la rápida difusión de las TIC asociada a la agenda de reforma en la gestión de las administraciones públicas, de hecho, muchos consideran esos cambios como fundamentales en las estructuras sociales y de gobierno de muchos países. De forma concreta, se ha definido gobierno electrónico como 'la aplicación de tecnologías basadas en Internet para actividades comerciales y no comerciales en el seno de las administraciones públicas' (Pocoví y Farabollini, 2002, p. 5).

El gobierno electrónico supone una nueva vía para entender cómo las administraciones públicas están rediseñando su organización y procedimientos, de manera que se puedan adecuar a un nuevo modelo de acción en el que el potencial de la información desempeña un papel central para la mejora de los servicios públicos. No debemos olvidar que el desarrollo del gobierno electrónico es más bien una cuestión política que de carácter técnico. De manera que Internet y las TIC pueden ser herramientas para el cambio, siempre que exista una clara voluntad política y directiva para liderar las transformaciones necesarias en las estructuras, procedimientos y cultura organizativa.

El gobierno electrónico permite que la organización se fundamente en el flujo de información más que en la jerarquía, implica operaciones racionalizadas y menos operadores intermedios o de base. Quienes dentro de las administraciones públicas se plantean proyectos de gobierno electrónico que van más allá de la información sobre la organización, se encuentran a menudo con importantes barreras y resistencias para realizarlos (sociales, corporativas, legislativas, procedimentales, inter-organizativas), siendo con frecuencia la tecnológica la menos importante. 
Por lo tanto en la implantación de la administración electrónica, como también se le denomina, deben intervenir productores de documentos, gestores del procedimiento y archivistas. Esto hace que cada archivo desarrolle algunos instrumentos, como por ejemplo, tablas de acceso y seguridad, con la finalidad de establecer los límites de acceso según la legislación vigente y los perfiles de usuarios que acuden en busca de información (Alvarez, 2007, p.13).

En lo específicamente administrativo, la masificación del documento electrónico, a través de su publicación utilizando tecnologías estándar de comunicación de datos, se debe ver como un paso indispensable para, a partir de la automatización de oficinas, avanzar hacia el gobierno electrónico. Sus grandes beneficios y los ahorros que genera, seguramente dan retorno a las inversiones. Sin embargo, su implementación en la administración pública requiere una serie de transformaciones que no pasan solamente por lo tecnológico o lo legal; el tendido de redes se debe hacer en el marco de una nueva cultura de la información (Pocoví y Farabollini, 2002, p.22).

Las iniciativas de gobierno electrónico y de promoción de la Sociedad de la Información deberán formularse teniendo en cuenta las especificidades de cada contexto social, político e institucional; contando con los actores críticos y los recursos necesarios que garanticen una adecuada definición de los problemas y la resolución efectiva de los mismos logrando los objetivos marcados. Es evidente que si el propio proceso de formulación de las políticas para la promoción de la Sociedad de la Información, en general, y los proyectos de gobierno electrónico, en particular, se realiza al margen de la población y de sus organizaciones, sin tener en cuenta su realidad y sus necesidades concretas, no será posible promover la apropiación social de Internet (Criado Grande; Ramilo Araujo y Salvador Serna, 2002, p.42).

La implementación del gobierno electrónico aspira a integrar la información y sus aplicaciones a través de una plataforma tecnológica común, accesos simples a los datos, facilidad de operación y vistas únicas de la información institucional. Estas nuevas tecnologías, al ser flexibles, pueden compartirse independientemente del ámbito laboral donde se desempeñe el usuario y de las características de su trabajo administrativo. Esta gobernabilidad será posible en la medida que la acción política, social y económica sea capaz de formularse de manera consensuada teniendo presentes las necesidades y demandas del conjunto de la sociedad para ofrecer respuestas y resultados adecuados que permitan resolver los problemas, cada vez más complejos, de manera efectiva. Sólo así será posible recuperar la legitimidad y la confianza en las instituciones públicas (Criado Grande; Ramilo Araujo y Salvador Serna, 2002, p.45).

Por lo tanto, es urgente emprender actividades de divulgación y promoción, alertar a los responsables de formular políticas y sensibilizar al gran público tanto sobre el potencial de los productos digitales como sobre los problemas prácticos que plantea su preservación. Dentro de este contexto es necesario destacar, la acción emprendida por la Conferencia General de la UNESCO, quien aprobó por unanimidad en sesión plenaria, el 10 de noviembre de 2010, la Declaración Universal sobre los Archivos, propuesta por el Consejo Internacional de Archivos (ICA), a raíz de una iniciativa de la Sección de Asociaciones Profesionales (SPA), que fue aprobado en la Asamblea General en Oslo en septiembre de 2010 (UNESCO Resoluciones, 2011, p.77).

La Declaración es una poderosa herramienta sobre la pertinencia de los archivos en la sociedad moderna, además pone énfasis en el rol que estos tienen como instrumento para la transparencia administrativa y rendición de cuentas democrática; así como, para la preservación de la memoria social.

Además, de no dejar de lado la tradicional preocupación por satisfacer las necesidades de la investigación histórica, la Declaración enfatiza la necesidad de una eficaz gestión de los archivos como una función esencial que apuntala no solo a la administración pública moderna sino también para fomentar buenas prácticas en la empresa privada, y de este modo facilitar el acceso a la información de los ciudadanos. Ahora, el reto es utilizar la Declaración para procurar el máximo efecto, por lo que los archivos deben salir del ghetto, en el que se encuentran todavía, a menudo, y ocupar su lugar como un actor importante en el corazón de la administración pública y el centro de la memoria social.

Los países que han tenido un esquema legal abierto a la libertad de información son los que disponen de una estructura más clara sobre el uso de la información, para que sea orientada a promover el libre acceso a la información y el impulso de las libertades públicas como era el objetivo inicial de la ley, sea con fines de 
instrumentalizar la información para hacerla accesible para otros fines orientados al bienestar de la ciudadanía o al impulso de una naciente industria de la información (Ramos, 2009, p.45).

La gestión efectiva de los archivos es una precondición para el buen gobierno, el estado de derecho, la transparencia administrativa, la preservación de la memoria colectiva de la humanidad y el acceso a la información por parte de los ciudadanos. Por lo que el papel de la Archivística debe ser concomitante al derecho interno e internacional, y para ello será necesario el establecimiento y desarrollo progresivo del diálogo entre éstos. La comunidad internacional de archivistas pueden servir de muchas formas a la humanidad, una de ellas es pugnar porque se dicten leyes con verdadero contenido científico-archivístico, y promover que a los archivos se les reconozca formalmente el carácter de garantes de los derechos humanos. La lucha contra la impunidad no puede ser consolidada sin archivos y sin archivistas, pero participar en esa lucha nos sitúa dentro de los sectores más vulnerables.

El papel de los archivistas como custodios de los archivos, en tanto garantes de los derechos humanos, no sólo los faculta si no que los obliga a participar activamente en todos los procesos concernientes a esos derechos. No existe democracia, estado de derecho, garantía efectiva ni ejercicio pleno de los derechos humanos, sin archivos, como testigos silenciosos de la verdad a los que el archivista tiene el deber de dar voz.

Los profesionales que "conocen" los documentos tienen criterio sobrado para delimitar cuando un documento es accesible o no, sin tener que esperar al legislador o al agente protector de datos. Los archivistas tienen formación y criterio suficiente para garantizar un acceso razonable a la documentación, pero también para limitarlo si es de justicia.

En la actualidad la profesión está en una encrucijada importante y que hay que plantearse fuertemente qué caminos hay que seguir, qué es estratégico para mejorar su posicionamiento social y cuáles deben ser los caballos de batalla de la innovación. El reto que suponen las nuevas tecnologías y la e-administración obliga a los profesionales a «controlar el documento desde que nace porque ya no tiene la dimensión física anterior, del papel, tanto para su uso normal como para su uso histórico», por lo que una separación orgánica como sucede en muchas partes entre Archivo Histórico/Cultura o Archivo Administrativo/Servicios Generales, no tiene sentido ni es operativa. Nos reduce posibilidades, cohesión y "divide" nuestros presupuestos restándonos competitividad.

Los archivistas, hoy en el siglo XXI, tendrán que hacer un gran esfuerzo y disponer de los documentos organizados. Sin olvidar que la complejidad de las organizaciones actuales necesita de un gran impulso de modernización. Haciendo uso de las telecomunicaciones y de la informática. Considerando la incorporación de medios electrónicos en el desarrollo de la actividad administrativa. Es cierto que durante estos años se han llevado a cabo serios intentos de sistematizar la administración y disponer de las TIC, de hecho en algunos casos, son ya una realidad en la propia gestión administrativa (Alvarez, 2007, p.11).

Con la adaptación de las tecnologías de la información y la comunicación en nuestro medio archivístico el trabajo del archivista no se limita a estar en su escritorio y entre los estantes. La labor de informar y transmitir conocimientos la debe ejercer en todas partes, y aunque a algunos no les parezca apropiado, debería realizarla en gran parte por fuera del archivo. Porque en el fondo, el deber es llevarle la información a quienes la necesitan y donde la necesitan, no esperar a que ellos vengan por ella al archivo. De ahí la importancia de la utilización adecuada de los recursos tecnológicos para la comunicación y la transmisión de información, no sólo para la gestión interna del archivo sino para la atención de los usuarios de una institución. Es muy importante tener bien claro que modernización no es tener muchos computadores ni automatizar todos los procesos. Esto es sólo parte de la modernización. Quizá la parte menos interesante. Lo fundamental es que todos los archivistas tengan conciencia de lo que implica la modernización para el desempeño de su labor. Y esto sólo se logra mediante un proceso de adaptación que incluye capacitación, instrucción, actualización permanente, participación activa en el planeamiento y en la toma de decisiones (Alvarez, 2007, p.15).

En la era de Internet la información pública es un activo en el sentido de que constituye un haber imprescindible para los ciudadanos en general y determinados grupos de la sociedad en particular. Si poseer información en formato impreso resultaba necesario para la toma de decisiones o el control de la gestión, ahora, en el entorno digital, gracias a su velocidad y capacidad para ser transmitida, procesada, combinada y/o desglosada de otras informaciones es ineludible tanto para los negocios o la política como para la vida cotidiana. Además, en el contexto 
de los poderes públicos toda la información generada, recibida o acumulada por ellos debe ser difundida a los ciudadanos, como medio de transparencia de sus actos, para permitir la participación de la ciudadanía en la vida pública y potenciar la confianza de la sociedad en las instituciones públicas que la sirven (Ramos, 2009, p.42).

Por todo ello, resulta evidente que un adecuado manejo de la información supone una clara mejora organizacional aunque no estén bien definidas ni las causas ni la amplitud de esa mejoría. Por lo tanto, una adecuada creación y manejo de la información del sector público son decisivos tanto por su valor en sí misma como por los activos económicos que representa.

En la mayoría de los países existe algún tipo de normativa que regula la gestión de los archivos. En la mayoría de los casos los sistemas de archivo encuentran su origen hace décadas -en algunos se remontan incluso al momento mismo de la creación del Estado- con un acento especial en el cuidado del acervo histórico y cultural, y como una manera de llevar un registro de bienes y recursos.

Existe una visión de la gestión de archivos como un área de menor importancia en general, que es desplazada como un aspecto poco relevante dentro de las funciones del Estado. Si bien se le asigna el rol de "conservar el acervo histórico y cultural", no se provee a estas instituciones de los medios normativos, humanos y económicos suficientes para poder llevar a cabo satisfactoriamente su tarea (la cual de por sí es ya demasiado genérica).

Al momento de la creación de estas primeras normas, la finalidad lejos estaba de concebir herramientas de archivo para la transparencia o accountability, por lo cual los temas que regula y el diseño institucional de aquellas no es apto para cubrir las necesidades actuales, propias de sistemas de acceso a la información y del rol activo de los ciudadanos. Por lo tanto, estas normas deben acompañarse de instituciones, llámese Archivo Nacional, Archivo General de la Nación o el Sistema Nacional de Archivos que dicten las políticas necesarias para el adecuado manejo del patrimonio documental (Informe Regional. 2011. p.27-28).

Un Archivo Nacional debe ser una organización sólida, con vocación transversal y no bipolarizada por la "Cultura/Historia/Archivística" o la "Administración de las organizaciones/Gestión Documental", por lo tanto, se debe demostrar que el patrimonio documental, el capital informativo, puede dar respuesta a los problemas del presente, ayudar a aportar criterio y capacidad crítica. La documentación se gestiona desde su fase inicial de producción y no se deja de preservar.

En cuanto a experiencias exitosas de puesta en práctica del gobierno electrónico es importante destacar el caso de Chile, donde este Programa mediante un instructivo presidencial propiciaba el uso de tecnologías de la información y la comunicación en los órganos de la administración del Estado para:

- mejorar los servicios de información ofrecidos a los ciudadanos,

- aumentar la eficiencia y eficacia de la gestión pública,

- incrementar la transparencia del sector público y la participación ciudadana.

Este programa de gobierno electrónico logró su mayor desarrollo conceptual y operativo a partir de la creación de la ley 19.799 sobre documentos electrónicos, firmas electrónicas y servicios de certificación de dichas firmas y la creación de un comité de normas encargado de establecer regulaciones para el uso de tecnologías en los servicios públicos. De acuerdo a lo anterior, entre otras cosas, se implementó un modelo de sistema más complejo, el que a partir del catálogo de documentos digitales y utilizando un flujo de trabajo automatizado, permite que el usuario pueda registrar su solicitud y cancelar los costos de los certificados en cualquier punto del país. 


\section{El acceso a la información}

El acceso a la información puede convertirse en una herramienta transformadora y de acceso a otros derechos fundamentales, como por ejemplo los derechos económicos, sociales y culturales. Por lo tanto, merece que atendamos el fortalecimiento de las buenas prácticas gubernamentales (Informe Regional. 2011. p.3).

Los archivos son frecuentemente considerados como "testigos silenciosos" especialmente cuando los Derechos Humanos están en juego, tienen un papel importante como testigo silencioso, como parte de la memoria y por ende como un instrumento de rendición de cuentas de lo que tuvo lugar en el pasado. Los archivos deben tenerse en cuenta en las acciones del gobierno de un modo auténtico y confiable, y para ello el acceso a esos archivos necesita garantizarse, naturalmente. En muchos países la "libertad de acceso a la información pública" de los ciudadanos es posible a través de la Ley de Archivos y/o de Acceso a la Información. Es importante no perder de vista que la Declaración Universal de los Derechos Humanos, ha inspirado legislaciones dirigidas a garantizar los derechos individuales y colectivos de la sociedad, estableciendo en su artículo $19^{\circ}$ el derecho a la información "por cualquier medio de expresión".

En adición a los conceptos tradicionales tales como capital físico y recursos -o capital- humanos, la noción de recursos de información se ha puesto al corriente en años recientes. Estos recursos incluyen no sólo información en la forma de archivos de datos y reportes programados, sino también sistemas que facilitan el acceso y la adquisición de información, que tienen valor en la toma de decisiones y otros procesos que contribuyen a los productos y servicios que la organización debe brindar a sus clientes.

La información es lo que nos gobierna en la actualidad y a la par de que se acrecienta en todas las áreas del conocimiento, surgen nuevos y mejores modos de transmitirla. Es importante estar preparados para asimilar los hechos, considerar que en todo esta gama de modernidad debemos adoptar lo que mejor nos convenga o se adapte a nuestro entorno y necesidades, entendiendo primeramente el efecto que esta herramienta puede tener en nuestra vida (González, 2005, p.531).

Desde el punto de vista de la prestación de servicios, la información dentro de las administraciones públicas puede desempeñar diferentes roles:

1. La información puede apoyar la gestión interna, que incluye información sobre el personal para la gestión de los recursos humanos, e información sobre el presupuesto o la contabilidad para la gestión financiera.

2. La información puede apoyar la regulación, que supone información que guarda los detalles de los principales actores y entidades dependientes, sean personas, empresas, edificios, tierra, importaciones/exportaciones.

3. La información puede apoyar los servicios públicos, información que difiere de acuerdo con el servicio público en particular, educación (archivos sobre el personal educador), sanidad (informes sobre los pacientes), o transporte (información sobre los movimientos de los pasajeros).

4. Finalmente, la información puede estar disponible públicamente, esto es, información que los gobiernos desean diseminar (informes de consultoría, detalles de políticas públicas o leyes y regulaciones), información que los gobiernos recopilan para luego darla a conocer (estadísticas demográficas o económicas), o información requerida para aprovisionarse (correspondencia, auditorías, documentos internos sobre políticas, respuestas a los ciudadanos, periodistas 0 políticos). (Pocoví y Farabollini, 2002, p. 31),

En cuanto a las características que ha de tener la información ofrecida a los ciudadanos encontramos:

- Información completa: sistematizada, protegida de accesos no deseados.

- Información objetiva, fiable, es decir, no sesgada presentada en función de su finalidad. 
- Información accesible, en distintos formatos y al alcance de sus destinatarios potenciales.

- Difusión proactiva, además de permitir el acceso, la Administración Pública debe dar publicidad a los documentos facilitando su conocimiento y localización.

- Acceso individual, la creación de canales individuales de comunicación, como Internet, propicia que la Administración favorezca el consumo individual de información.

- Información de calidad, que cumpla estándares técnicos. Los requisitos técnicos de calidad, se incorporan como una exigencia de la accesibilidad de la información; si ésta no cumple con unas características técnicas formales es como si no existiera.

- Relevante, plantea la necesidad de que la información esté sometida a un tratamiento, técnico y de contenido, de manera que los ciudadanos la encuentren fácilmente.

- Fácil de encontrar, integrada, organizada.

- Fácil de usar: la información es un derecho y un bien económico que goza de la característica de bien público, de tal manera que el uso por unos, no excluye a los demás (Ramos, 2009, p.43).

En estos enunciados aparecen ya algunos de los aspectos más controvertidos de los nuevos problemas generados por la información creada y gestionada por los poderes públicos: protección de los datos personales y de la intimidad, organización de la información en función de la finalidad de su utilización, costes en su recogida y tratamiento, como modo de impedir que el Estado se convierta en un gran Leviatán. Por lo tanto, se deben definir algunos criterios para garantizar el derecho de acceso a la información pública, entre los que podemos encontrar:

- La voluntad política del Estado para implementar una verdadera cultura de transparencia.

- La infraestructura, tecnología, gestión y organización adecuada de toda entidad pública obligada a brindar información.

- La capacitación adecuada de la población y de los funcionarios públicos (responsables o no de brindar información).

- La autonomía e independencia en el ejercicio de funciones del órgano garante del derecho de acceso a la información pública. Sus funcionarios (acceso, nombramiento, ascenso y permanencia).

- Un sistema normativo que establezca de manera clara un mandato pleno para que el órgano garante pueda decidir, disponer medidas correctivas e incluso establecer sanciones. Asimismo, que establezca un procedimiento de acceso a la información pública ágil, accesible, conocido y amigable.

Muchos países aún no cuentan con leyes de acceso a la información. Si bien en algunas naciones se ha avanzado con políticas de transparencia que se aplican sólo al poder ejecutivo, estos esfuerzos muestran serias limitaciones. El fracaso de esos intentos da cuenta de la imperiosa necesidad de que los países que aún no cuentan con una ley de acceso a la información, construyan un marco legal en el cual se sustenten los ciudadanos para hacer valer sus derechos.

La puesta en vigencia de una ley de acceso a la información pública, ha impactado por ejemplo, los procesos internos y los servicios ofrecidos por el Archivo Nacional de Chile, en donde las tecnologías de la información y la comunicación han si do utilizadas como herramientas de apoyo para una mejor implementación de dicha ley. La puesta en vigencia de la ley de acceso a la información pública más que generar grandes cambios en los procesos y servicios fundamentales de los archivos, viene a plantear como principal desafío el hacer eficiente el acceso a la información en los tiempos y formas que hoy en día se requieren, para lo cual, el uso de las TIC resultan indispensables y estratégicas (González, 2010, p.7). 
El advenimiento de los estados democráticos y participativos enfocados en el desarrollo de políticas inclusivas, como es el caso chileno, demanda un interés mayor por temáticas que aborden políticas de información, dirigidas a la ciudadanía y al establecimiento de la democracia.p.7 Por lo tanto, es importante la definición de políticas de información que pueden ser entendidas como, un conjunto de mecanismos, orientaciones y de directrices utilizadas por las sociedades para la aplicación, el control de los ciclos de vida tanto de la información como del documento, así como el ciclo de transferencia de los datos / información y documento.

Una política de información es "una decisión gubernamental, que regula todas las actividades del sector y es el resultado de una correlación de fuerzas dentro del ámbito del Estado" mas también, como agrega Lindoso, la institucionalización de una política pública de información efectiva, debe involucrar actores sociales (comités constituidos por diferentes instancias: órganos gubernamentales, financiadores, cuerpos efectivos, funcionarios, usuarios y productores de bienes culturales) que representen todas las expresiones del área Informacional (Sá Xavier de Lira y Marques de Oliveira, 2005, p.7).

El monitoreo de políticas que involucran la generación, uso y transferencia de información es una tarea de sujetos políticos que ejercen un monitoreo autónomo, de ahí que los derechos fundamentales del ciudadano imponen una nueva postura por parte del Estado, que en el gerenciamiento del patrimonio público debe dar cuentas a la sociedad, siendo transparente, socialmente responsable y políticamente correcto, y principalmente garantizando la efectividad del control social ciudadano.

El acceso es la disponibilidad de los documentos para ser consultados como resultado tanto de una autorización legal como de la existencia de instrumentos de descripción. Si se acepta que el objeto de la Archivística son los archivos, los documentos y la información en ellos contenida, debidamente organizados para la consulta y la buena conservación de los mismos en una sociedad democrática, todos los ciudadanos deben acceder a la documentación e información, al patrimonio documental, que recibe y genera la administración ya sea esta un municipio, un estado, un departamento, un país, según sea el caso, que en definitiva están a su servicio. Para ello es necesario, entre otras cosas, que dicha administración tenga sus archivos en condiciones de accesibilidad, pues de lo contrario, no se pueden consultar, este objetivo es tan antiguo como la organización civil de la humanidad, los documentos han sido creados y conservados por necesidades del gobierno y administración, en consecuencia, la gestión del poder y la gestión de los documentos están estrechamente ligados. Desde 1994 el Consejo Internacional de Archivos (CIA) ha publicado cuatro normas sobre la descripción archivística: ISAD (G) en 1994, ISAAR (CPF) en 1996, ISDF en el 2008, e ISDIAH en el mismo año. Estas normas abarcan los instrumentos de descripción, uno de los dos elementos clave en el acceso a los archivos y que han transformado la práctica de la descripción (Alvarez, 2007, p.10).

El Consejo Internacional de Archivos ha generado dos documentos adicionales que subrayan la importancia del acceso como un elemento de la práctica archivística: el Código de Ética de 1996 y la Declaración Universal de Archivos de 2010, citada en párrafos anteriores. El Código de Ética declara en los principios 6 y 7 :

- Principio 6: Los archivistas deberán promover el acceso más amplio posible a los materiales archivísticos y proporcionar un servicio imparcial a todos los usuarios.

- Principio 7: Los archivistas deberán respetar tanto el acceso como la privacidad, y actuar dentro de los límites de la legislación pertinente. (CIA. Código de Etica, 1996, pp. 2-3)

Por su parte, la Declaración Universal de Archivos destaca, la necesidad vital que tienen los archivos de apoyar la eficiencia administrativa, responsabilidad y transparencia, para proteger los derechos de los ciudadanos, para establecer la memoria individual y colectiva, para comprender el pasado, y para documentar el presente afín de guiar futuras acciones. Así como, identifica uno de los roles vitales de los archivistas que es: hacer posible que los documentos estén disponibles para su uso, y los compromete a que trabajen juntos a efectos de que: los archivos sean accesibles para todos, respetando las leyes pertinentes y los derechos de las personas, productores, dueños y usuarios.

El propósito fundamental de los archivos es su uso. El servicio de acceso vincula los archivos con el público; determina la información que los usuarios tienen acerca de la institución y su acervo; influye en la confianza que el público deposita en los custodios de las instituciones archivísticas y en el servicio que ellos proporcionan. 
El grupo de trabajo sobre el acceso del Comité de buenas prácticas y normas del Consejo Internacional de Archivos formuló en el año 2011 unos principios de acceso a los archivos. Los Principios de Acceso a los Archivos están compuestos por 10 principios, con un comentario que explica cada uno de ellos; los Principios y los comentarios tomados en conjunto constituyen la declaración de la práctica profesional, que sigue a la presentación del Código de Ética. Los principios están acompañados por orientaciones técnicas y un breve glosario.

Entre los alcances de estos principios encontramos que:

a) Abarcan tanto los derechos de acceso del público como las responsabilidades de los archivistas para posibilitar el acceso a archivos y a la información sobre ellos.

b) Reconocen que la administración del acceso puede implicar una restricción al mismo, basado en la información que contienen los archivos.

c) Reconocen, además, que en algunos países diversas leyes codifican las reglas de acceso que resultan inconsistentes entre sí. Esto es cierto tanto para leyes que rigen los archivos bajo custodia el gobierno, como para leyes relativas a archivos privados, de organismos no gubernamentales.

Las responsabilidades primarias por la implementación de estos principios son compartidas por los administradores, archivistas, donantes y por el personal de las instituciones que transfieren sus documentos para la custodia archivística. Dentro de los principios destacaremos los siguientes:

1. El público tiene el derecho de acceso a los archivos de los organismos públicos. Tanto las entidades públicas como privadas deben abrir sus archivos en la medida de lo posible. El acceso a los archivos de gobierno resulta esencial para una sociedad informada. La democracia, la responsabilidad, el buen gobierno y el compromiso cívico requieren de una garantía legal respecto de que las personas tendrán acceso a los archivos de organismos públicos establecidos por gobiernos nacionales, territorios autónomos, gobiernos locales, instituciones intergubernamentales, y cualquier organización o persona natural o legal que desempeñen funciones públicas y que operen con fondos públicos. Todos los archivos de organismos públicos están abiertos al público salvo que se encuadren en una excepción prevista en la ley.

2. Las instituciones que custodian archivos deben dar a conocer la existencia de archivos, incluyendo la existencia de documentos no consultables, y revelar la existencia de restricciones que afectan el acceso a los archivos. Por lo tanto, los usuarios deben ser capaces de ubicar la institución archivística que custodia el material de su interés.

Las instituciones que posibilitan el acceso al público a cualquier parte de sus archivos deben divulgar la política de acceso. Los usuarios tienen el derecho de saber si existe una serie específica, archivo, documento o parte del mismo cuyo uso esté vedado.

3. Las instituciones que custodian archivos deben adoptar un enfoque proactivo sobre el acceso.

Los archivistas tienen la responsabilidad profesional de promover el acceso a los archivos, por lo tanto, deben utilizar varios medios para hacer conocer los archivos, incluyendo Internet y publicaciones en la página web, materiales impresos, programas públicos, medios comerciales y otras actividades de divulgación. Además, deben estar continuamente alertas a los cambios tecnológicos de comunicación

4. Las instituciones que custodian archivos deben asegurar que las restricciones de acceso son claras y tienen una duración determinada, basadas en la legislación pertinente, en el conocimiento del derecho de privacidad, de acuerdo con las normas culturales, y el respeto de los derechos de los propietarios de los documentos privados. Los archivistas deben proporcionar el mayor acceso posible a los archivos, pero también deben reconocer y aceptar la necesidad de algunas 
restricciones. Las restricciones son impuestas por la legislación o por la política institucional, por la institución archivística o su organismo rector, o por el donante. En última instancia, las restricciones van en contra del propósito social de preservación archivística respecto de la responsabilidad y el patrimonio, y los archivistas deben procurar limitar el alcance de las restricciones a la información que verdaderamente necesita de la retención temporal.

5. Los archivos deben estar disponibles en términos igualitarios de acceso. Los archivistas deben proporcionar a los usuarios un acceso a los archivos justo, equitativo y oportuno. Dado que, personas de diferentes categorías utilizan los archivos, las reglas de acceso deben diferenciar entre las distintas categorías de usuarios (por ejemplo, público en general, personas adoptadas que buscan información sobre el nacimiento de sus padres, investigadores médicos buscando información estadística sobre registros hospitalarios, víctimas de violación de los derechos humanos), pero las reglas deben aplicarse igualitariamente a todas las personas, dentro de cada categoría sin discriminación( Principios de acceso a los archivos. 2011. p.9-11).

Al formular una política escrita de acceso se deben establecer las bases sobre las que se toman las decisiones sobre el acceso. La política de acceso debe hacer referencia a cualquier ley, orden o decisión judicial, regulaciones y acuerdos con el donante que se aplican a los archivos.

Las leyes deben incluir una ley específica sobre los archivos; libertad de información, privacidad y protección de datos, y actos privados; legislación que incluye acceso general o disposiciones sobre el secreto; y leyes que se aplican a los documentos del sector privado como ser legislación en las corporaciones, instituciones educativas, y escribanos.

La política de acceso describe las restricciones específicas y generales que se aplican a los archivos. Las restricciones generales se aplican a determinados tipos de información o a clases designadas de material, dondequiera que se encuentren en los archivos. Las restricciones específicas son detalladas por la entidad que lo transfiere y se aplican sólo a los archivos de un productor o donante determinado. La política de acceso incluye una referencia genérica a restricciones específicas para alertar a los usuarios sobre la existencia de las mismas, si bien la inclusión de cada restricción específica podría crear una política engorrosa y confusa lo que usualmente no es recomendado.

\section{El caso de Costa Rica}

En Costa Rica aún no se ha generado una ley sobre transparencia, quizás porque desde el año 1990 se promulgó la Ley No. 7202 del Sistema Nacional de Archivos, la que procuró integrar en su articulado algunos temas relacionados con el acceso, que encuentra su mecanismo jurídico, en el recurso de Amparo previsto por la Ley de Jurisdicción Constitucional, por violación de los artículos 27 y 30 de la Constitución Política, derecho que puede utilizar cualquier persona que pretenda acceder a información pública. El recurso de amparo ante la Sala Constitucional es el mecanismo para resolver los conflictos entre particulares y el Estado en materia de acceso a la información. Es una petición que puede presentar cualquier ciudadano o ciudadana sin necesidad de patrocinio letrado y sin ningún tipo de formalidad: el ciudadano o ciudadana la dirige a la Sala Constitucional, la que solicita informes al funcionario responsable, quien debe de rendirlo en un plazo de tres días hábiles. Una vez recibido el informe, la Sala se pronuncia (Informe Regional. 2011. p.15).

En la Asamblea Legislativa, se encuentran algunos proyectos de ley que se ubican en los últimos lugares de la agenda que tienen que ver con esta temática, muchos de ellos ya fueron archivados, a saber: Ley para garantizar la transparencia en la administración de justicia pública y privada, Adicción de unos párrafos al artículo 4 de la Ley General de Administración Pública sobre transparencia institucional y resguardo efectivo del derecho de petición, el derecho a la información, la rendición de cuentas y el deber de probidad, Creación del sistema general de transparencia y rendición de cuentas en el estado costarricense, Ley de transparencia y acceso a la información pública, Ley de acceso a la información para la transparencia en la gestión pública, Ley de idoneidad, transparencia y ética en la función pública y El derecho a la información. (Proyectos de Ley. Asamblea Legislativa) El hecho de que no sean prioritarios para los congresistas revela el poco interés que revierte esta temática para los tomadores de 
decisiones, pese a que el impulso dado al gobierno electrónico debe ir de la mano con asuntos como el acceso a la información, la transparencia, la rendición de cuentas, etc.

En la última década se han dictado algunas leyes que en alguna medida la vienen a complementar este derecho constitucional, como es el caso de la Ley No. 8220 de Protección al ciudadano del exceso de requisitos y trámites administrativos de 4 de marzo de 2002 y su Reglamento, la Ley General No. 8292 de Control Interno del 31 de Julio del 2002 y su Reglamento, la Ley No. 8454 de Certificados, Firmas Digitales y Documentos Electrónicos de 30 de agosto de 2005 y su Reglamento, y las Normas técnicas para la gestión y el control de las tecnologías de la información de 21 de junio de 2007.

Por otra parte, con motivo de la promulgación de la Ley de certificados, firmas digitales y documentos electrónicos, el Archivo Nacional, tomando como referencia el Dictamen C-283-98 de la Procuraduría General de la República, emitió la Directriz con las regulaciones técnicas sobre la administración de los documentos producidos por medios automáticos, publicada en la Gaceta No. 221 del jueves 11 de noviembre del 2004 y de acatamiento obligatorio en el Sistema Nacional de Archivos, para la gestión de documentos producidos por medios automáticos (los documentos creados en un ambiente electrónico o que se conserven en un soporte electrónico), que deberá ser aplicada por el jerarca, titulares subordinados, encargados de archivos centrales y encargados de tecnologías de información, y que deja sin efecto la anterior, a la que se han sumado una serie de circulares.(Circulares. Dirección General del Archivo Nacional) Estas últimas, entre otras cosas han procurado que se no se pierda de vista que el ente rector en materia archivística es el Archivo Nacional, poniendo énfasis en el hecho de que como conocedor de la realidad archivística de las instituciones públicas, a través de los informes archivísticos que éstas deben verter anualmente; se ha detectado que gran cantidad de documentos, esenciales para una administración eficiente y transparente del Estado y parte de los cuales además poseen valor científico-cultural, se producen y conservan sin ninguna normalización ni prevención, con lo cual no se garantiza su permanencia en el tiempo y por consiguiente se corre el riesgo de perder información indispensable para una adecuada administración de las instituciones y eventualmente la pérdida del patrimonio documental de nuestro país, en diferentes soportes. Por ello se permitió recordar, entre otras cosas, algunos artículos, de las principales normativas que sigue en esta materia:

El artículo 3 de la Ley del Sistema Nacional de Archivos considera de valor científico cultural aquellos documentos textuales, manuscritos, o impresos, gráficos, audiovisuales y legibles por máquina, que por su contenido, sirvan como testimonio y reflejen el desarrollo de la realidad costarricense.

El artículo 11, incisos e), f), h) de la Ley № 7202 del Sistema Nacional de Archivos, señala que le corresponde a la Junta Administrativa del Archivo Nacional las funciones de:

- "Establecer las políticas archivísticas del país y recomendar estrategias para un adecuado desarrollo del Sistema Nacional de Archivos.

- Formular recomendaciones técnicas sobre la producción y la gestión de documentos.

- Formular recomendaciones técnicas sobre la administración de documentos producidos por medios automáticos."

El artículo 6 del Reglamento de la Ley del Sistema Nacional de Archivos ordena a la Junta Administrativa del Archivo Nacional formular las políticas archivísticas del país y recomendar las estrategias para el adecuado desarrollo del Sistema Nacional de Archivos.

El artículo 6 de la Ley de Certificados, Firmas Digitales y Documentos Electrónicos y su Reglamento, vigente desde el 21 de abril del 2006, en lo relativo al Estado y sus instituciones establece que en gestión y conservación de documentos electrónicos se aplicará la Ley del Sistema Nacional de Archivos No. 7202, en cuanto a dictar las regulaciones necesarias para asegurar la gestión de vida y conservación de los documentos, mensajes o archivos electrónicos. 
La Ley General de Control Interno No. 8292, en el artículo 16 establece la obligación de los jerarcas y titulares subordinados de contar con sistemas de información que permitan a la administración activa tener una gestión documental institucional.

Además, dentro del ordenamiento jurídico costarricense se encuentran una serie de leyes que han sido reformadas con el fin de tipificar el delito informático relacionado con gestión y acceso a la información.

No hay que perder de vista que como lo señala el Consejo Internacional de Archivos, un documento es una información que ha sido producida o recibida en la ejecución, realización o término de una actividad institucional o personal y que engloba el contenido, el contexto y la estructura permitiendo probar la existencia de esa actividad. Para tener un valor probatorio, un documento debe ser considerado confiable y auténtico. La confiabilidad está ligada al hecho de que el documento está autorizado y es digno de fe (es decir que corrobora los hechos). La autenticidad se refiere a que el documento es lo que pretende ser. Esas dos cualidades dependen del contenido, de la estructura y del contexto en el que se genera el documento, según lo establece el ordenamiento jurídico vigente.

Los encargados de los archivos centrales deben participar activa y eficientemente en la consecución de sistemas de información que permitan a la administración activa tener una gestión documental institucional, por lo que como se hiciera mención en párrafos anteriores, una adecuada gestión documental es fundamental para garantizar la transparencia administrativa, el derecho de acceso a la información y pronta respuesta y la rendición de cuentas.

En varias instituciones se ha tratado de resolver los problemas de información solamente desde la perspectiva informática y no de manera integral, evidenciando la existencia de una separación entre las áreas de la Informática y la Archivística que genera una inadecuada gestión y conservación documental.

Los documentos conservados en medios automáticos son más susceptibles a la degradación por razones de polvo, campos magnéticos, temperatura, humedad, deformaciones del soporte y otros. Además, gran cantidad de documentos son generados, procesados y cumplen su propósito en medios electrónicos sin garantizar su autenticidad, integridad y confiabilidad.

La garantía de permanencia (conservación) de un documento en soporte electrónico es un problema que no ha podido ser resuelto, ni siquiera con los últimos avances de la tecnología. Existen medios de almacenamiento para los documentos producidos por medios automáticos, que garantizan la permanencia de estos por largos períodos y sin embargo, no garantizan que muchos años después existirá un dispositivo que pueda leer la información en esos medios, impidiendo el acceso a la información (Directriz con las regulaciones técnicas sobre la administración de los documentos producidos por medios automáticos.2008. p.4).

Como se desprende de lo anteriormente señalado, el Archivo Nacional, siempre ha mostrado preocupación porque se respete el ordenamiento jurídico en materia archivística, asumiendo el papel que le corresponde como ente rector del Sistema Nacional de Archivos, velando por una adecuada gestión de documentos y por un uso racional de las tecnologías de la información y la comunicación; máxime que como se analiza a continuación, se han producido algunas acciones alrededor de la puesta en marcha del gobierno electrónico, lo que ha impulsado la producción de documentos en soporte electrónico en forma desmedida, sin tomar en cuenta aspectos tan importantes como son, el adecuado control en la concepción, producción, mantenimiento, utilización y conservación de los documentos producidos por medios automáticos. Dado que, en última instancia lo que interesa es que se pueda garantizar su autenticidad, integridad y confiabilidad, ya que como parte del patrimonio documental en soporte digital, podría correr el peligro de perderse para la posteridad, a lo que contribuyen, entre otros factores, la rápida obsolescencia de los equipos y programas informáticos que le dan vida, las incertidumbres existentes en torno a los recursos, la responsabilidad y los métodos para su mantenimiento y conservación y la falta de legislación que ampare estos procesos (Carta para la preservación del patrimonio digital. 2003. p.80).

Dentro de este marco no podemos dejar de lado las acciones de la Presidencia de la República, quien en el año 2006, emitió el decreto 33147 por medio del cual se crea la Comisión Intersectorial de Gobierno Digital y la Secretaría Técnica de Gobierno Digital como instrumento instructor que entre otros objetivos planteaba: 
a) Incrementar la transparencia y el acceso a la información gubernamental.

b) Facilitar los mecanismos de ciudadanía activa en la interacción con el Estado.

c) Incentivar la eficiencia en la Administración Pública por medio del uso de tecnologías digitales.

La Estrategia Gobierno Digital tiene por objeto contribuir, mediante el aprovechamiento de las Tecnologías Digitales, a la construcción de un Estado más eficiente, más transparente, más participativo y que preste mejores servicios a los ciudadanos y las empresas, lo cual redunda en un sector productivo más competitivo, una administración pública moderna y una comunidad más informada y con mejores instrumentos para la participación. Estos aspectos nos dan la base para la definición de los ejes de acción que rigen la estrategia de gobierno digital, a saber:

- Calidad de los servicios

- Transparencia y participación

- Eficiencia del Estado

- Acceso

- Gestión y Políticas (Eje de la Secretaría Técnica de Gobierno Digital) (Plan de acción. p.15)

En cuanto a la implementación del gobierno electrónico, éste se conceptualiza como gobierno digital y su estrategia se desarrolla en cuatro áreas de acción: Gobierno a Ciudadano (G2C): Proporciona servicios orientados al ciudadano. Gobierno a Negocio o Empresa (G2B): Proporciona servicios rápidos y transparentes. Gobierno a Gobierno (G2G): Gobierno interconectado y eficiente. Infraestructura: Infraestructura favorable para las TIC y marco legal.

Todo ello con la finalidad de responder en forma planificada y controlada a las necesidades propias conducentes a la consolidación de una sociedad de la información para Costa Rica (Plan de acción. Gobierno digital. 2008-2010. p.6).

Para la puesta en marcha de la estrategia, se han planteado unas líneas de acción alrededor de las cuales se han articulado algunos proyectos, a saber, en cuanto a calidad de servicios se han propuesto los siguientes proyectos: Portal y centro de datos, Portal comercialización y encadenamiento de Mipymes, Sistema integrado de expediente de salud. En la línea de Transparencia, se ha propuesto: Permisos de extranjería, Ciudades digitales, Voto electrónico, Teletrabajo, Capacitación en línea. En la de Eficiencia del Estado, se han propuesto: Modernización del sistema de emisión de cédulas de identidad, Automatización del cobro de tasas de peajes, Acueductos y Alcantarillados Digital y Publicación de actos administrativos (Leyes y decretos). En cuanto a Acceso, encontramos: Infraestructura de conectividad del Estado costarricense, Bibliotecas virtuales, Conectando ideas, Fomento y certificación de Cafés Internet y Cruz Roja digital. Por último, en la línea de Gestión y políticas, se han propuesto los siguientes proyectos: Implementación de la oficina de administración de proyectos para el Sector Público y Monitoreo e-gobierno (Plan de acción. Gobierno digital. 2008-2010. pp.17-21).

De todas las acciones antes mencionadas y dada su relación con el patrimonio digital, merece destacarse el proyecto Presidencia Digital de Costa Rica, según el cual se digitalizará todo el proceso de ingreso, revisión y envío de Decretos a la Imprenta Nacional, en forma conjunta con la Dirección de Leyes y Decretos de Presidencia, pues se argumenta que en este momento si un Ministerio emite un decreto, debe enviarlo físicamente a Presidencia, firmarlo y enviarlo a la Imprenta o devolverlo al Ministerio si hubiera alguna anotación, para que inicie de nuevo el proceso.

Con este proyecto que se ya se encuentra en ejecución, las entidades tienen dos opciones: enviar vía correo electrónico o a través de la nueva intranet que se creó para estos fines, en ambos casos utilizando la firma digital. En la Presidencia harán las observaciones bajo las mismas vías y darán la aprobación con el uso de la firma digital. 
Entre los principales beneficios del proyecto se señalan el uso de tecnología, la eliminación de papel y la disminución del reproceso, lo que producirá que los trámites se lleven a cabo con mayor rapidez. Este proyecto pertenece a la Estrategia Nacional de Simplificación de Trámites que impulsa el actual Gobierno dentro del marco del Gobierno Digital, sin embargo se han dejado de lado, consideraciones de tipo archivístico especialmente en cuanto al adecuado control en la concepción, producción, mantenimiento, utilización y conservación de este tipo de documentos, producido por medios automáticos. De manera tal que, se pone en evidencia que la Estrategia Gobierno Digital, no ha tomado en cuenta la importancia que reviste el patrimonio digital, máxime en el caso de documentos que tienen un valor permanente y como se ha señalado; asimismo factores como, la rápida obsolescencia de los equipos y programas informáticos que le dan vida, las incertidumbres existentes en torno a los recursos, la responsabilidad y los métodos para su mantenimiento y conservación.

\section{Conclusión}

Del análisis anterior se puede concluir que pese a que las tecnologías de la información y la comunicación están plenamente incorporadas a nuestro quehacer diario, en el sector público se han tomado algunas decisiones apresuradas en cuanto a su incorporación para la generación de documentos en otros soportes especialmente lo que conocemos como documentos electrónicos, incorporados dentro de los planes del gobierno digital, como se le denomina en Costa Rica. Por esta razón, merece destacarse el papel que ha asumido el Archivo Nacional que bajo el amparo de algunas políticas de la UNESCO y de acciones emprendidas por el Consejo Internacional de Archivos ha tratado de crear conciencia en la Administración Pública de la importancia de prestar atención a los documentos que se están generando en soportes no tradicionales y que al final pueden llegar a formar parte del patrimonio digital.

La problemática del patrimonio digital como se ha puesto en evidencia no solo tiene que ver con tratamiento y conservación sino también con lo relativo al acceso a la información, al que tienen derecho todos los ciudadanos pero que se puede ver lesionado por la ausencia de un marco jurídico adecuado, de lo que existen innumerables testimonios en otras latitudes, máxime si están de por medio las tecnologías de la información y la comunicación especialmente en cuanto a la obsolescencia y a la posibilidad de acceso que puedan tener los usuarios de la información que generan las instituciones, custodiada en unidades de información como son los archivos. Por lo que sirva esta reflexión para crear conciencia sobre la importancia de emprender acciones inmediatas para evitar que el patrimonio digital pueda estar disponible para las generaciones futuras, en la que se deben involucrar todos los sectores especialmente a nivel gubernamental. 


\section{Referencias}

Alianza regional. Por la Libre Expresión e Información. (2011) Informe Regional del Estado de Situación de Acceso a la Información Pública $143^{\circ}$ Período de Sesiones de la Comisión Interamericana de Derechos Humanos. Washington, Estados Unidos. 1-36 Recuperado el 03 de febrero de 2012, de http://www. es.scribd.com/doc/70140327

Alvarez Rodríguez, Mariela. (2007). Los archivos del hoy, patrimonio documental del mañana: una propuesta para la organización técnica de los documentos en los archivos. VII Congreso de Archivología del Mercosur. Viña del Mar, Chile, 1-15

Añorve Guillén, Martha Alicia. Archivos y sociedad. (2007). Revista General de Información y Documentación. 17, núm. 2.123-128

Consejo Internacional de Archivos. Comité de Buenas Prácticas y Normas. Grupo de Trabajo sobre el Acceso. Principios de Acceso a los Archivos. Versión Preliminar. 2011, 1-37

Criado Grande, J. Ignacio; Ramilo Araujo, María Carmen y Salvador Serna, Miquel. (2002). La Necesidad de Teoría(s) sobre Gobierno Electrónico. Una Propuesta Integradora. XVI Concurso de Ensayos y Monografías del CLAD sobre Reforma del Estado y Modernización de la Administración Pública "Gobierno Electrónico". Caracas, Venezuela, 1-52

Delgado Gómez, Alejandro. (2010). Documentos y poder: órdenes del discurso. Anales de Documentación, 13, 117-133.

Dirección General del Archivo Nacional. (2006) CIRCULAR. Dirigida a: Archivistas costarricenses. Asunto: Ley No 8454 "Ley de Certificados, Firmas digitales y Documentos electrónicos" e Informe Archivístico Anual. San José, Costa Rica. Recuperado el 10 de diciembre de 2011, de http://www.archivonacional.go.cr

Dirección General del Archivo Nacional. (2005) CIRCULAR: Oficio DG-1013-2005. Dirigida a: Jerarcas de Ministerios, Municipalidades e Instituciones Descentralizadas. Asunto: Ley № 7202 del Sistema Nacional de Archivos y el patrimonio documental. San José, Costa Rica. Recuperado el 10 de diciembre de 2011, de http://www.archivonacional.go.cr

Dirección General del Archivo Nacional. (2008). Directriz con la regulaciones técnicas sobre la administración de los documentos producidos por medios automáticos. San José, Costa Rica,1-7 Recuperado el 10 de diciembre de 2011, de http://www.archivonacional.go.cr

González G., Eliana. (2010). Soluciones tecnológicas para el acceso y uso de la información de los archivos. 3er Seminario internacional de archivos de tradición. De la Transparencia a los Archivos : el derecho de acceso a la información ibérica. Santiago, Chile, 1-14

González Meneses, Ofelia Isabel. La sociedad de la información: ventajas y desventajas. (2005). Memoria. XXXV Jornadas Mexicanas de Biblioteconomía. Asociación Mexicana de Bibliotecarios, A.C. Cancún, Quintana Roo, México, 531-540

Grupo de trabajo sobre el acceso. Comité de buenas prácticas y normas. (2011). Principios de Acceso a los Archivos. Versión Preliminar. Consejo Internacional de Archivos. París, Francia

International Council on Archives. (1996). Code of Ethics. Beijing, China

Organización de las Naciones Unidas para la Educación, la Ciencia y la Cultura. (2003) Actas de la Conferencia General. Resoluciones. $32^{\mathrm{a}}$ reunión. Volumen 1. París, Francia.

Organización de las Naciones Unidas para la Educación, la Ciencia y la Cultura. (2011) Actas de la Conferencia General. Resoluciones. Volumen 1. París, Francia.

Plan de Acción. Gobierno Digital. Costa Rica 2008-2010. Recuperado el 05 de enero de 2012, de http://www.gobierno fácil.go.cr

Pocoví, Gertrudis María Estela y Farabollini, Gustavo Ricardo. (2002). Gobierno electrónico: un cambio estructural. La integración de la información como requisito. XVI Concurso de Ensayos y Monografías del CLAD sobre Reforma del Estado y Modernización de la Administración Pública "Gobierno Electrónico". Caracas, Venezuela, 1-29

Procuraduría General de la República. (1998). Dictamen C-283-98. San José, Costa Rica.

Proyectos de ley. Asamblea Legislativa de Costa Rica. Recuperado el 03 de febrero de 2012, de http://www.asamblea.go.cr

Ramos Simón, Luis Fernando; Mendo Carmona, Concepción y Arquero Avilés, Rosario. (2009). La producción informativa y documental del Estado: hacia un inventario de los recursos públicos. Revista Española de Documentación Científica. 32, 1, eneromarzo, 40-59

Sá Xavier de Lira, Aquiles y Marques de Oliveira, Jemima. (2005). Política pública de informação na pespectiva do controle social na ciência da informação: o caso do programa é pra ler da Prefeitura Municipal de João Pessoa. Biblionline, v. 1, n. 2, 1-15 
Biblios La problemática del patrimonio digital: El caso de Costa Rica

\section{Datos del autor}

José Bernal Rivas Fernández

Profesor catedrático de la Universidad de Costa Rica. Magister en Scienta en Historia. Subdirector de la Escuela de Historia. Coordinador de la Sección de Archivística.

jose.rivas@ucr.ac.cr

Recibido-Received : 2012-02-14

Aprobado-Accepted : 2012-09-29

\section{(cc) EY-NC-ND}

This work is licensed under a Creative Commons

Attribution-Noncommercial-No Derivative Works 3.0 United States License.

\section{ULIS D-Sunf}

This journal is published by the University Library System of the University of Pittsburgh as part of its $\underline{D-S c r i b e}$ Digital Publishing Program and is cosponsored by the University of Pittsburgh Press. 